\title{
Hydration in hemolytic uremic syndrome
}

\author{
Manuel D. Bilkis, M.D. ${ }^{a}$ and Pablo Bonany, M.D. ${ }^{b}$
}

\begin{abstract}
Diarrhea-associated hemolytic uremic syndrome is preceded by gastroenteritis due to Shiga toxin-producing Escherichia coli. Support measures are recommended, specifically, fluid restriction to avoid cardiopulmonary overload. However, in the prodromal period or with established hemolytic uremic syndrome, volume expansion with isotonic fluids is safe and effective, and reduces the need for dialysis, the length of hospital and intensive care stay, neurological events, and hyponatremia.

Therefore, when nephrological monitoring is available and / or short-term access to a tertiary care hospital is guaranteed, it is suggested to hydrate patients withnosigns of cardiopulmonary overload, regardless of their renal function, with initial volume expansion. Afterwards, if an adequate urine output is achieved, the patient should not be dialyzed (except if they have a medically intractable metabolic/electrolyte disorder) and hydration should be continued with an isotonic solution containing $5 \%$ dextrose for adequate hydration and urine output.

Keywords: hemolytic uremic syndrome, dehydration, fluid therapy, extracellular fluids.
\end{abstract}

http:/ / dx.doi.org/ 10.5546/ aap.2021.eng.62

To cite: Bilkis MD, Bonany P. Hydration in hemolytic uremic syndrome. Arch Argent Pediatr 2021;119(1):6266.

a. Emergency

Department.

b. Department of

Nephrology.

Hospital de Niños

"Ricardo Gutiérrez,"

Autonomous City

of Buenos Aires,

Argentina.

E-mail address:

Manuel D. Bilkis, M.D.: mbilkis@gmail.com

Funding:

None.

Conflict of interest:

None.

Received: 7-19-2020

Accepted: 9-15-2020

\section{INTRODUCTION}

Shiga toxin-producing Enterobacteriaceae, especially Shiga toxin-producing E. coli (STEC), are responsible for causing systemic damage through thrombotic microangiopathies. This may affect several organs, such as the liver, pancreas, skin, heart, brain, and particularly the kidneys. In addition to microangiopathy, dehydration and lower renal blood flow may worsen prognosis in this condition known as hemolytic uremic syndrome (HUS). ${ }^{1}$

Diarrhea-associated hemolytic uremic syndrome (D+HUS) is defined as the presence of gastroenteritis followed by thrombocytopenia (less than 150000 platelets $/ \mathrm{mm}^{3}$ ), microangiopathic hemolytic anemia (hematocrit below $30 \%$ ), and acute renal failure with blood creatinine levels above the upper limit for age. $\mathrm{D}+\mathrm{HUS}$ has no specific treatment. In much of the world, E. coli O157: H7 is the major cause of D+HUS.

Support measures are recommended for treatment, which traditionally include fluid restriction to avoid cardiopulmonary fluid overload in the acute phase. ${ }^{2}$ This volume restriction measure should be reviewed considering the correlation between dehydration, low isotonic fluid intake, and poor course in HUS.

\section{Hemolytic uremic syndrome and dehydration}

The progression from STEC infection to HUS, which occurs in $10-15 \%$ of cases, used to be assumed as an unavoidable fatality. HUS can be categorized as either oligoanuric or non-oligoanuric. Children with oligoanuric HUS generally require dialysis, have more complicated courses, and have a higher risk for chronic sequelae. However, it was not known whether the events that occurred early in STEC infections, particularly dehydration and measures to expand circulating volume, affected the likelihood of experiencing oligoanuria and acute renal failure in D+HUS.

J. A. Ake et al. ${ }^{3}$ retrospectively compared 2 groups of patients with $\mathrm{D}+\mathrm{HUS}$, an oligoanuric group and a non-oligoanuric group, and observed that the volume and sodium content of intravenous fluids administered early in illness had affected the risk for developing oligoanuric HUS after E. coli O157:H7 infections. Patients with oligoanuric HUS had received less fluid volumes and sodium at the beginning of the disease and had a 
poorer course: a greater percentage of patients who required dialysis and a longer length of stay in the hospital. The authors proposed that patients with bloody diarrhea (BD) due to STEC would benefit from intravascular volume expansion with isotonic solutions at $20 \mathrm{~mL} / \mathrm{kg}$ plus intravenous maintenance according to basal requirements, before D+HUS is diagnosed and develops. ${ }^{3}$ C. A. Gianantonio et al. ${ }^{4}$ described a better long-term prognosis in patients with shorter initial oligoanuria.

Initial dehydration in D+HUS is a predisposing factor for an increased prevalence of dialysis, as described by Balestracci et al. ${ }^{5}$ who reviewed the data of 137 children with HUS and divided them into two groups according to their hydration status at admission: normally hydrated (n: 86) and dehydrated (n: 51). The dehydrated group had a higher need for dialysis $(70.6 \%$ versus $40.7 \%, p=0.0007) . .^{5}$ They concluded that dehydration at hospital admission might represent a concomitant factor aggravating the intrinsic renal disease in D+HUS patients. Therefore, they encouraged the early recognition of patients at risk of D+HUS to guarantee a wellhydrated status with isotonic fluids.

J. M. Ojeda et al. ${ }^{6}$ studied 36 patients with $\mathrm{D}+\mathrm{HUS}$; 21 of them required dialysis (58\%; $95 \%$ confidence interval [CI]: 40.8-75.8) and 13 (36.1\%; $95 \%$ CI: 19.0-53.1) did not attain a complete recovery of renal function. In a model of bivariate analysis, the only significant risk factor was dehydration, defined as the loss of more than $5 \%$ of body weight (odds ratio [OR]: 5.3; $95 \% \mathrm{CI}$ : $1.4-12.3 ; p=0.0220)$. In a multivariate model (Cox regression), dehydration was marginally significant (hazard ratio: 95.823; $95 \%$ CI: 93.175109.948; $p=0.085)$. Results suggested that dehydration before hospital admission might increase the risk for an incomplete recovery of renal function in the long term in children who had D+HUS.

In patients with D+HUS, Ardissino et al. ${ }^{7}$ retrospectively analyzed the presence of factors associated with dialysis and/or neurological involvement. This group proposed that hemoconcentration and hypovolemia, associated with dehydration, may be responsible for more severe ischemia and organ damage both in the short and long term. At disease onset, these signs should be considered risk factors for a more severe course. Therefore, they recommended that hydration status should be actively monitored in HUS patients and that dehydration should be promptly corrected. ${ }^{7}$

In a retrospective, multicenter, observational study from our setting, L. Alconcher et al. ${ }^{8}$ reported a mortality rate of $3 \%$ in D+HUS, which was statistically related to hyponatremia, hemoconcentration, and / or central nervous system (CNS) involvement. Other authors described combinations of initial ancillary tests in D+HUS which determined that dehydration was a predictor of poor course..$^{8-12}$

\section{Hemolytic uremic syndrome and hydration}

The search of medical care for children with a suspected or confirmed STEC infection before HUS occurs is a potential opportunity to mitigate subsequent renal failure. Hickey et al. ${ }^{13}$ conducted a prospective, multicenter, observational study on the effects of isotonic volume expansion in patients with diarrhea due to STEC before developing D+HUS. Out of 50 participants, $68 \%$ were oligoanuric. It is striking that, in the group of patients who did not receive intravenous fluids (without expansion), in the first 4 days of illness, the oligoanuric rate was $84 \%$. The researchers suggested that intravenous volume expansion (VE) was an underused intervention that could decrease the frequency of oligoanuric renal failure in patients at risk of HUS. ${ }^{13}$

D. Loconsole et al. ${ }^{14}$ established an operating protocol for BD in a pediatric population as a rapid response to a public health threat represented by an excess of pediatric D+HUS cases in the Apulia region (Italy) starting from 2013. Positive STEC cases underwent vigorous VE. Of them, $7.5 \%$ evolved into HUS, all with favorable outcome.

In a multicenter study conducted in 38 children's hospitals from the United States and Canada, R. McKee et al. ${ }^{15}$ studied 927 STECinfected children; $41(4.4 \%)$ had HUS at presentation; of the remaining 886, 126 (14.2\%) developed HUS. Predictors of HUS included younger age (OR: 0.77; 95 \% CI: 0.69-0.85/ year), leukocyte count $\geq 13.0 \times 10^{3} / \mu \mathrm{L}$ (OR: 2.54; $95 \%$ CI: 1.42, 4.54), higher hematocrit (OR: 1.83; $95 \%$ CI: 1.21, 2.77/5\% increase), higher serum creatinine (OR: 10.82; $95 \%$ CI: $1.49,78.69 / 1 \mathrm{mg} / \mathrm{dL}$ increase), platelet count $<250 \times 10^{3} / \mu \mathrm{L}$ (OR: 1.92; $95 \%$ CI: 1.02, 3.60), lower serum sodium (OR: 1.12; $95 \%$ CI: 1.02, $1.23 / 1 \mathrm{mmol} / \mathrm{L}$ decrease), and intravenous fluid administration initiated $\geq 4$ days following diarrhea onset (OR: 2.50; $95 \%$ CI: 1.14, 5.46). The identified risk factors highlighted the importance 
of avoiding dehydration through early VE in $\mathrm{BD}$ and performing close clinical and laboratory monitoring.

G. Ardissino et al. ${ }^{16}$ hydrated patients with isotonic solutions at D+HUS onset until reaching a weight $10 \%$ greater than the reference working weight. The objective was to restore circulating volume and reduce ischemic or hypoxic tissue damage. The short- and long-term outcomes of these patients were compared with a group of historical patients, from a period when the indicated treatment was fluid restriction. Patients had significantly better short-term outcomes with a lower rate of CNS involvement $(7.9 \%$ versus $23.7 \%, p=0.06$ ), had less need for dialysis $(26.3 \%$ versus $57.9 \%, p=0.01)$ or a shorter length of stay at the intensive care unit (2.0 versus 8.5 days, $p=0.02$ ), and needed fewer days of hospitalization (9.0 versus 12.0 days, $p=0.03$ ). Long-term outcomes were also significantly better in terms of renal and extrarenal sequelae $(13.2 \%$ versus $39.5 \%, p=0.01)$. In this way, this group concluded that patients with D+HUS had great benefit from early VE. They also suggested that early and generous fluid infusions may reduce thrombus formation and ischemic organ damage, thus having positive effects on both short- and long-term disease outcomes. ${ }^{16}$

In adults, an outbreak of D+HUS was studied, for which VE was used as an early strategy before the clinical manifestation of the disease, and $30 \%$ of cases required dialysis $(5 / 15),{ }^{17}$ which was lower than the $54 \%(160 / 298)$ observed in another outbreak where VE was not used. ${ }^{18}$

Recently, the Pediatric Nephrology ${ }^{19}$ published a retrospective study that we conducted, in which 35 D+HUS patients were analyzed: 16 received VE with isotonic fluids and 19 were patients who received conventional fluid restriction (FR). Neither group presented evidence of fluid overload upon admission or during treatment. Only $12.5 \%$ of patients in the VE group required replacement therapy versus $47.4 \%$ in the FR group. In addition, VE corrected initial hyponatremia, and serum sodium was maintained within normal ranges

Regarding neurological complications and mortality, fewer or no events were observed in the VE group, although they did not show statistical differences, which is probably related to the small size of the sample. ${ }^{19}$ For this reason, when nephrological monitoring is available and / or short-term access to a tertiary care hospital is guaranteed, it is suggested to initially hydrate
D+HUS patients with no signs of fluid overload, regardless of the status of their renal function, by infusing a $0.9 \%$ saline solution at $10 \mathrm{~mL} / \mathrm{kg} / \mathrm{h}$ over a 3-hour period. Afterwards, if urine output is higher than $0.5 \mathrm{~mL} / \mathrm{kg} / \mathrm{h}$, the patient should not be dialyzed (except if they have a medically intractable metabolic or electrolyte disorder) and hydration should be continued, according to their needs, with an isotonic saline solution containing $5 \%$ dextrose for 48 hours, in order to maintain an adequate hydration and urine output (Table 1).

\section{Volume expansion in hemolytic uremic syndrome}

C. Ahn et al..$^{20-22}$ advocate for VE in the BD stage due to Shiga toxin-producing Enterobacteriaceae: first with a $0.9 \%$ saline solution expansion at $20 \mathrm{~mL} / \mathrm{kg}$ and then with maintenance fluids with potassium if its concentration is normal or low. This therapy is continued until symptoms improve or platelets are maintained above $150000 / \mathrm{mm}^{3}$. A daily blood count monitoring is required, including platelet count, electrolytes, blood urea, and blood creatinine.

S. Grisaru et al. ${ }^{23}$ conducted a review and metaanalysis on intravenous fluid administration, hydration status, and progression before developing D+HUS. They found that a hematocrit value greater than $23 \%$ as a measure of dehydration status at presentation with HUS was associated with the development of oligoanuric HUS (OR: 2.38 [95 \% CI: 1.30-4.35]; I ${ }^{2}=2 \%$ ), need for renal replacement therapy (OR: 1.90 [95\% CI: $1.25-2.90] ; \mathrm{I}^{2}=17 \%$ ), and death (OR: 5.13 [95\% CI: 1.50-17.57]; $\mathrm{I}^{2}=55 \%$ ). Intravenous fluid administration up to the day of HUS diagnosis was associated with a decreased risk for renal failure and dialysis (OR: 0.26 [95 \% CI: 0.11-0.60]). ${ }^{23}$

\section{Diarrhea-associated hemolytic uremic syndrome and hyponatremia}

Hyponatremia is a common initial presentation in patients diagnosed with $\mathrm{D}+\mathrm{HUS}$ (between $30 \%$ and $50 \%$ of cases, according to published series). Two concomitant conditions are its pathophysiological root. First, a strong non-osmotic stimulus to antidiuretic hormone, as a consequence of dehydration, vomiting, anemia, intestinal inflammation, and abdominal pain; and second, the attempts to hydrate patients by oral administration of hypotonic solutions during the prolonged and progressive prodromal period. Hyponatremia is related to CNS complications, such as seizures and encephalopathy, and is 
TABLE 1. Volume expansion protocol

1) Inclusion criteria: every patient diagnosed with D+HUS, who is normotensive, with a normal cardiac silhouette on chest $\mathrm{X}$-ray and no signs of fluid overload, regardless of their renal function.

2) Dose: infusion of a $0.9 \% \mathrm{NaCl}$ solution at $10 \mathrm{~mL} / \mathrm{kg} / \mathrm{h}$ over a 3-hour period. Afterwards, if the patient achieved a urine output higher than $0.5 \mathrm{~mL} / \mathrm{kg} / \mathrm{h}$, treatment should be continued with an isotonic saline solution (130-154 mEq/L) containing $5 \%$ dextrose according to basal requirements, as per the Holliday-Segar formula: 0-10 kg, $100 \mathrm{~mL} / \mathrm{kg} /$ day; $10-20 \mathrm{~kg}$, $50 \mathrm{~mL} / \mathrm{kg} /$ day; and more than $20 \mathrm{~kg}, 20 \mathrm{~mL} / \mathrm{kg} /$ day.

also a strong predictor of death risk in D+HUS patients. ${ }^{24-27}$

In our study, the initial hyponatremia was corrected in 24 hours, and blood sodium was kept within normal ranges in the VE group. After 48 hours, the number of patients with hyponatremia was statistically and significantly higher in the FR group $(p=0.014){ }^{19}$

\section{CONCLUSIONS}

VE with isotonic fluids is safe and effective in patients with diarrhea due to Shiga toxinproducing Enterobacteriaceae and once the HUS diagnosis is established. It improves and mitigates HUS progression.

\section{REFERENCES}

1. Gianantonio C, Vitacco M, Mendilaharzu F, Ruty A, et al. The hemolytic-uremic syndrome. J Pediatr. 1964; 64(4): 478-91.

2. Grisaru S. Management of hemolytic-uremic syndrome in children. Int J Nephrol Renovasc Dis. 2014; 7:231-9.

3. Ake JA, Jelacic S, Ciol MA, Watkins SL, et al. Relative nephroprotection during Escherichia coli O157:H7 infections: association with intravenous volume expansion. Pediatrics. 2005; 115(6):e673-80.

4. Gianantonio CA, Vitacco M, Mendilaharzu F, Gallo G. The hemolytic-uremic syndrome. Renal status of 76 patients at long-term follow-up. J Pediatr. 1968; 72(6):757-65.

5. Balestracci A, Martin SM, Toledo I, Alvarado C, et al. Dehydration at admission increased the need for dialysis in hemolytic uremic syndrome children. Pediatr Nephrol.2012; 27(8):1407-10.

6. Ojeda JM, Kohout I, Cuestas E. La deshidratación al ingreso es un factor de riesgo para la recuperación incompleta de la función renal en niños con síndrome urémico hemolítico. Nefrología. 2013; 33(3):372-6.

7. Ardissino G, Daccò V, Testa S, Civitillo CF, et al. Hemoconcentration: a major risk factor for neurological involvement in hemolyticuremic syndrome. Pediatr Nephrol. 2015; 30(2):345-52.

8. Alconcher LF, Coccia PA, Suarez ADC, Monteverde ML, et al. Hyponatremia: a new predictor of mortality in patients with Shiga toxin-producing Escherichia coli hemolytic uremic syndrome. Pediatr Nephrol. 2018; 33(10):1791-8.

9. Ardissino G, Tel F, Testa S, Paglialonga F, et al. A simple prognostic index for Shigatoxin-related hemolytic uremic syndrome at onset: data from the ItalKid-HUS network. Eur J Pediatr. 2018; 177(11):1667-74.

10. Balestracci A, Meni Battaglia L, Toledo I, Martin SM, et al. Comment to: "A simple prognostic index for Shigatoxinrelated hemolytic uremic syndrome at onset: data from the ItalKid-HUS network" by Ardissino et al. Eur J Pediatr.
2018 Aug 10. doi: 10.1007/ s00431-018-3198-7. Eur J Pediatr. 2018; 177(12):1869-70.

11. Keenswijk W, Vanmassenhove J, Raes A, Dhont E, et al. Blood urea nitrogen to serum creatinine ratio is an accurate predictor of outcome in diarrhea-associated hemolytic uremic syndrome, a preliminary study. Eur J Pediatr. 2017; 176(3):355-60.

12. Balestracci A, Meni Battaglia L, Toledo I, Martin SM, et al. Blood urea nitrogen to serum creatinine ratio as a prognostic factor in diarrhea-associated hemolytic uremic syndrome: a validation study. Eur J Pediatr. 2018; 177(1):63-8.

13. Hickey CA, Beattie TJ, Cowieson J, Miyashita Y, et al. Early Volume Expansion During Diarrhea and Relative Nephroprotection During Subsequent Hemolytic Uremic Syndrome. Arch Pediatr Adolesc Med. 2011; 165(10):884-9.

14. Loconsole D, Giordano M, Laforgia N, Torres D, et al. Case-management protocol for bloody diarrhea as a model to reduce the clinical impact of Shiga toxin-producing Escherichia coli infections experience from Southern Italy. Eur J Clin Microbiol Infect Dis. 2020; 39(3):539-47.

15. McKee R, Schnadower D, Tarr P, Xie J, et al. Predicting Hemolytic Uremic Syndrome and renal replacement therapy in Shiga Toxin-producing Escherichia coli Infected children. Clin Infect Dis. 2020; 70(8):1643-51.

16. Ardissino G, Tel F, Possenti I, Testa S, et al. Early Volume Expansion and Outcomes of Hemolytic Uremic Syndrome. Pediatrics. 2016; 137(1):e20152153.

17. Sanders T, Ellis G, Castrovinci P, Deiss R, et al. 1098. Clinical Features and Outcomes of United States Marine Corps Recruits Hospitalized With Shiga Toxin-Producing Escherichia coli Infection and Hemolytic-UremicSyndrome. Open Forum Iinfect Dis. 2018; 5(Suppl 1):S329-30.

18. Menne J, Nistchke M, Stinngele R, Abu-Tair M, et al. Validation of treatment strategies for enterohaemorrhagic Escherichia coli O104:H4 induced haemolytic uraemic syndrome: case-control study. BMJ. 2012; 345:e4565.

19. Bonany P, Bilkis MD, Iglesias G, Braun A, et al. Fluid restriction versus volume expansion in children with diarrhea-associated HUS: a retrospective observational study. Pediatr Nephrol. 2020 Jul17. [Online ahead of print].

20. Ahn CK, HoltN, Tarr P.Shiga-Toxin Producing Escherichia coli and the Hemolytic Uremic Syndrome: What Have We Learned in the Past 25 Years? Adv Exp Med Biol. 2009; 634:1-17.

21. Holtz R, Neill M, Tarr P. Acute Bloody Diarrhea: A Medical Emergency for Patients of All Ages. Gastroenterology. 2009; 136(6):1887-98.

22. Davis TK, McKee R, Schnadower D, Tarr P. Treatment of Shiga Toxin-Producing Escherichia coli Infections. Infect Dis Clin North Am. 2013; 27(3):577-97.

23. Grisaru S, Xie J, Samuel S, Hartling L, et al. Associations Between Hydration Status, Intravenous Fluid Administration, and Outcomes of Patients Infected With Shiga Toxin-Producing Escherichia coli. A Systematic Review and Meta-analysis. JAMA Pediatr. 2017; 171(1): 68-76. 
66 / Arch Argent Pediatr 2021;119(1):62-66 / Special article

24. Freedman S, Grisaru S, Xie J, Samuel Set al. Management of Shiga toxin producing Escherichia coli-infected children: A multi-national, multi-specialty survey. J Paediatr Child Health. 2018; 54(4):390-7.

25. Bilkis MD, Montero D, VicenteF, Cheistwer A. Hidratación endovenosa en la práctica clínica. Nuevos enfoques terapéuticos para la gastroenteritis aguda. Arch Argent Pediatr. 2007; 105(5):436-43.
26. Feld LG, Neuspiel DR, Foster BA, Leu MG, et al. Clinical Practice Guideline: Maintenance Intravenous Fluids in Children. Pediatrics. 2018; 142(6):e20183083.

27. Kamioka I, Yoshiya K, Satomura K, Kaito H, et al. Risk factors for developing severe clinical course in HUS patients: a national survey in Japan. Pediatr Int. 2008, 50(4):441-6. 